\title{
MOTOR PROGRAMS: AN ARTIFICIAL NEURAL NETWORK APPROACH
}

\author{
W.K.T. Hau, I.C. Bruce, L.Y.L. Siu \&*E.Y.H. Chen. Department of Physiology and \\ *Department of Psychiatry, \\ Faculty of Medicine, The University of Hong Kong, Hong Kong
}

\begin{abstract}
:
It is commonly assumed that, during learning, the brain creates "motor programs" which store all the information essential to performing a motor skill. Yet there is still no consensus on what constitutes a motor program. In this study, a Multilayer Perceptron (MLP) network with one hidden layer, trained using the backpropagation rule, was used in an attempt to identify motor programs. Nine healthy subjects were asked to use their left hand to make fast and accurate movements in a tracking task of 75 identical steps, by either wrist flexion and extension, or the precision grip. The electromyogram (EMG) activity of 8 finger and hand muscles were simultaneously recorded by standard techniques. Onset timing of muscle activities were quantified from the digitized EMG signals, and were then used as the inputs to the MLP network. Reaction time was also measured, providing the desired output of the network. The trained network captured salient features of the relationship between EMG onset times and reaction time.
\end{abstract}

Key Words:

Motor Program, Artificial Neural Network, Multilayer Perceptron, Backpropagation Rule.

\section{Introduction}

Although the theortical existence of a motor program has long been accepted by researchers, from experimental psychologists [1] and motor behaviorists [2] to neurophysiologists [3], there is still no otjective measure of what a motor program is, of how and where it is created.

In 1968, Keele defined a motor program as 'a set of muscle commands that are structured before a movement sequence begins, and that allows the entire sequence to be carried out.' These muscle commands include the onset time, force-time envelope, and offset time for each muscle contributing to the movement [1]. Keele also proposed that each motor program was specific to a particular motor task.

Artificial neural networks have been used in a wide variety of biomedical signal-processing contexts [4-6]. What is novel in our approach is the use of an artificial neural network to identify components of motor programs. The network is trained to reproduce the input-output relationship from the data by repeatedly sifting the data back to the network, and correcting the network's error by adjusting the weights. The weights embody all of the information extracted during training.

One of the most widely used artificial neural networks is the multilayer perceptron (MLP) which can be trained by an algorithm called backpropagation. Backpropagation is a gradient-descent algorithm that minimizes the average squared error between the network outputs and the desired outputs. The most commonly used MLP architecture is a threelayer network, which contains an input layer, a hidden layer, and an output layer. Data is fed into the network through the input layer. The hidden layer contains processing elements (PEs) where intermediate internal processing takes place, and the strengths of the inter-layer connections (weights) are generated; while the desired output is fed back to the network via the output layer (Fig. 1).

In this study, we employed the MLP to investigate motor programs in terms of the temporal patterns of EMGs when subjects performed simple motor skills.

\section{Materials \& Methods:}

Subjects:

Nine healthy, right-handed volunteers ( 5 male, 4 female, 26-30 years old) participated in the experiment. They were naïve with regard to the specific purpose of the study. All experimental procedures were approved by the the University of Hong Kong Ethics Committee. 
Experimental Setup:

Each subject was seated comfortably in a dental chair, with the forearms resting on an adjustable support. An individually-fitted thermoplastic cast held the left forearm in the same posture over the recording period. Participants were asked to perform two tasks: 1. Subjects grasped a force transducer (Medical Research Ltd., Leeds UK) between index finger and thumb of the left hand and repeated precision grips; and 2. Subjects controlled a manipulandum (bearing a potentiometer to record the position changes) and performed wrist extensions and flexions. Surface electrode pairs were placed about $1 \mathrm{~cm}$ apart on the skin overlying the muscles: Abductor Digiti Quinti (ADQ), Abductor Pollicis Brevis (APB), First Dorsal Interosseous (FDI), Flexor Carpi Radialis (FCR), Flexor Carpi Ulnaris (FCU), Extensor Carpi Radialis (ECR), Extensor Carpi Ulnaris (ECU) and Extensor Digitorum Communis (EDC).

Approximately $60 \mathrm{~cm}$ in front of the participant, an oscilloscope screen displayed a cursor and a target. For task 1, the cursor was a horizontal trace that moved in proportion to the grip force; it moved upward or downward as grip force increased or decreased, respectively. For task 2 , the cursor was a horizontal trace that moved in proportion to the wrist movement; upward with wrist extension, and downward with wrist flexion. In both tasks, the target was a horizontal trace, which also moved in the vertical axis; the location of the target on the oscilloscope screen was determined by the computer.

Tasks:

At the beginning of the study, the subjects were asked to superimpose the cursor line on the target line. The target then jumped to a new position. The subject was required to move the cursor as accurately, and as fast as possible, to this new position. After $500 \mathrm{~ms}$, the target then moved to back to the initial position and the subjects were asked to move the cursor to follow the target movement, as accurately and as fast as possible, back to the initial position. The above sequence was repeated 75 times for both tasks. No practice trials were allowed.

Data collection:

While the subjects performed the step-tracking movements, the output of the force transducer or potentiometer and EMG activities were stored in computer memory. The raw EMG signals were amplified (x1000) and band-pass filtered $(30 \mathrm{~Hz}-$ $1 \mathrm{kHz}$ ) using conventional preamplifiers (Model P15, Grass Instruments), and then digitized with 12-bit resolution at a sampling rate of $1 \mathrm{kHz}$ (DT21EZ, Data Translation Inc.).

Data analysis:

Reaction time (RT) was determined by the time from the onset of the target movement to the onset of cursor movement. EMG signals were full-wave rectified, and the onset times of muscle activity were detected by visual inspection.

Network parameters:

The topology of the network we chose was a 3layer MLP with one hidden layer containing 8 PEs. EMG onset times provided the input to the network, while RT was the desired output for the network. The stop criterion chosen was less than $0.05 \%$ error. The hyperbolic tangent function was used as the activation function.

Through the interactive presentation of input patterns, with known desired outputs, the error between the predicted output from the network and the desired output was minimized by applying the backpropagation rule. Training continued until the error reached the stop criterion of $0.05 \%$. Weights with positive values meant the associated inputs had 'excitatory' effects on determining the desired output, while weights with negative values meant the associated inputs had 'inhibitory' effects on determining the desired output. So, during training, a more positive or negative weight meant the associated inputs had greater influences on determining the desired output. In contrast, inputs with lesser influences on the desired output tended towards zero. By examining the weights, we identified the key inputs which had the largest influence on determining the desire output. After each training sequence, the learning rate and the resulting weights were recorded for further analysis.

\section{Results:}

Our results showed that the time of activity onset for all the muscle studied, and the 
parameter RT;, became more or less constant after about 50 trials, i.e. the subjects had learned the task. We therefore assumed that the timing relationships of the 8 muscles during the remaining 25 trials constituted the subjects' "learned motor programme."

In order to answer the question regarding the objective features of motor programs, the muscle onset times and the measured RTs from the last 25 trials of all the subjects were used to train an MLP for each task using the same initial weights. The results showed that, with the same initial weights, the learning rates were the same for both tasks, and the final weights of the trained networks were remarkably similar (Table 1).

\section{Discussion:}

The precision grip and wrist flexion/extension are two different movements involving different prime movers operating at different joints. The precision grip involves a complex multi-joint and multi-muscle system, while the wrist movement only involves a single joint moved by agonist and antagonist muscles. So, how do the temporal features of the two motor programs differ? The results suggest that the grouped subjects used almost the same temporal components of the motor program to perform the two tasks.

The final weights of the trained MLP represent the input-output relationship between the muscles' onset timings and the measured RTs, and thus reflect one of the parameters in Keele's hypothetical definition of a motor program. Our results also favor Keele's suggestion that each motor program was specific to a particular motor task. In this study, although the subjects were using two different movements to achieve the same goal, the learning rates and the final weights of both trained neural networks were very similar, so if the final weights reflect the temporal properties within a motor program, all the subjects are using a similar motor program in both tasks. Further experiments will include additional features such as offset times and EMG amplitude measures.

\section{Conclusions:}

This preliminary study shows artificial neural networks can be used in a new approach to investigate motor programs and how they are implemented in motor control.

References:

[1] Keele SW: Movement Control in Skilled Motor Performance. Psychol. Bull. 70, 387-404, 1968.

[2] Cater MC and Shapiro: Control of Sequential Movements: Evidence for Generalized Motor Programs. Journal of Neurophysiology, Vol.52(5), 1984.

[3] Sanes JN and Jennings VA: Centrally Programmed Patterns of Muscle Activity in Voluntary Motor Behavior of Humans. Exp. Brain Res., Vol.54, 23-32, 1984.

[4] Kocur CM, Rogers SK, Myers LR, Burns T, and Kabrisky M: Using Neural Networks to Select Wavelet Features for Breast Cancer Diagnosis. IEEE EMB Mag. 15(5), 95-102, 1996.

[5] Kamangar FA and Behbehani K: An Articial Neural Network-Based Controller for the Control of Induced Paralysis Using Vecuronium Bromide. Annals of Biomedical Engineering, Vol.25, 1040-1051, 1997.

[6] Kothari $\mathrm{R}$, Cualing $\mathrm{H}$ and Balachander $\mathrm{T}$ : Neural Network Analysis of Flow Cytometry Immunophenotype Data. IEEE Trans. Biomedical Engineering, Vol.43(8), 803-809, 1996. 


$\begin{array}{lrrrrrrrr}\text { Table.1A } & \text { PE1 } & \text { PE2 } & \text { PE3 } & \text { PE4 } & \text { PE5 } & \text { PE6 } & \text { PE7 } & \text { PE8 } \\ \text { ADQ } & 0.18 & 0.16 & 0 & 0.54 & -0.44 & -0.49 & 0.36 & 0 \\ \text { APB } & 0.42 & 0.12 & 0.43 & -0.1 & -0.02 & 0.23 & -0.4 & -0.1 \\ \text { FDI } & -0.08 & 0.52 & 0.35 & 0.52 & 0.25 & 0.34 & -0.23 & 0.06 \\ \text { FCR } & 0.33 & -0.05 & -0.34 & 0.22 & -0.13 & 0.1 & -0.24 & -0.07 \\ \text { FCU } & 0.1 & -0.11 & -0.3 & -0.1 & 0.22 & -0.53 & 0.17 & -0.36 \\ \text { ECR } & 0 & 0.33 & -0.25 & 0.59 & -0.27 & -0.09 & -0.16 & 0.26 \\ \text { EDC } & -0.4 & -0.01 & 0.31 & 0.1 & -0.29 & 0.24 & 0.5 & 0.36 \\ \text { ECU } & 0.16 & -0.06 & -0.43 & 0.41 & 0 & -0.3 & 0 & -0.6 \\ & & & & & & & & \\ \text { Table.1B } & \text { PE1 } & \text { PE2 } & \text { PE3 } & \text { PE4 } & \text { PE5 } & \text { PE6 } & \text { PE7 } & \text { PE8 } \\ \text { ADQ } & 0.2 & 0.19 & 0 & 0.55 & -0.44 & -0.55 & 0.36 & 0 \\ \text { APB } & 0.43 & 0.13 & 0.42 & -0.1 & -0.02 & 0.19 & -0.4 & -0.1 \\ \text { FDI } & -0.07 & 0.54 & 0.34 & 0.51 & 0.25 & 0.3 & -0.23 & 0.07 \\ \text { FCR } & 0.33 & -0.05 & -0.34 & 0.18 & -0.15 & 0.1 & -0.24 & -0.05 \\ \text { FCU } & 0.1 & -0.11 & -0.3 & -0.15 & 0.21 & -0.52 & 0.17 & -0.32 \\ \text { ECR } & 0 & 0.34 & -0.26 & 0.56 & -0.29 & -0.1 & -0.16 & 0.27 \\ \text { EDC } & -0.4 & 0 & 0.3 & 0.08 & -0.3 & 0.22 & 0.5 & 0.37 \\ \text { ECU } & 0.16 & -0.05 & -0.44 & 0.38 & -0.02 & -0.31 & 0 & -0.6\end{array}$

Table.1

Table 1A shows the weight of each processing element (PE) associated with inputs to the MLP network trained by data from the wrist movement. Table $1 \mathrm{~B}$ shows the weight of each $\mathrm{PE}$ associated with inputs to the MLP network trained by data from the precision grip. The learning rate for both networks was the same.

Fig.1 A diagram shows the architecture of a Multi-Layer Perceptron neural network (MLP).

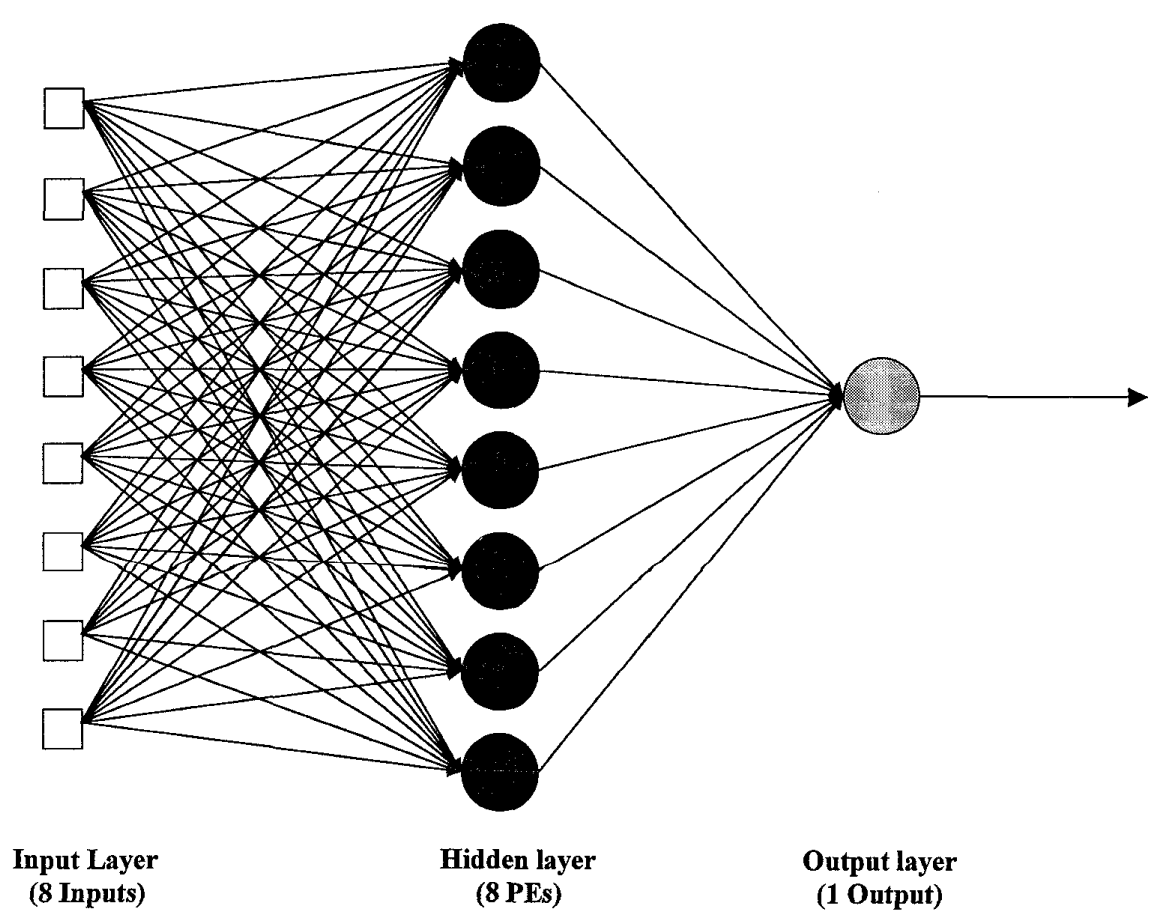

